\title{
Polymer physics predicts the effects of structural variants on chromatin architecture
}

\author{
Simona Bianco $\odot 1,9$, Darío G. Lupiáñez $\oplus^{2,3,4,8,9}$, Andrea M. Chiariello ${ }^{1,9}$, Carlo Annunziatella1,9, \\ Katerina Kraft ${ }^{2,3}$, Robert Schöpflin ${ }^{5}$, Lars Wittler 6 , Guillaume Andrey ${ }^{(2)}{ }^{2}$, Martin Vingron ${ }^{(5}{ }^{5}$, \\ Ana Pombo ${ }^{7}$, Stefan Mundlos ${ }^{2,3,4 *}$ and Mario Nicodemi ${ }^{1 *}$
}

\begin{abstract}
Structural variants (SVs) can result in changes in gene expression due to abnormal chromatin folding and cause disease. However, the prediction of such effects remains a challenge. Here we present a polymer-physics-based approach (PRISMR) to model 3D chromatin folding and to predict enhancer-promoter contacts. PRISMR predicts higher-order chromatin structure from genome-wide chromosome conformation capture (Hi-C) data. Using the EPHA4 locus as a model, the effects of pathogenic SVs are predicted in silico and compared to $\mathrm{Hi}-\mathrm{C}$ data generated from mouse limb buds and patient-derived fibroblasts. PRISMR deconvolves the folding complexity of the EPHA4 locus and identifies SV-induced ectopic contacts and alterations of 3D genome organization in homozygous or heterozygous states. We show that SVs can reconfigure topologically associating domains, thereby producing extensive rewiring of regulatory interactions and causing disease by gene misexpression. PRISMR can be used to predict interactions in silico, thereby providing a tool for analyzing the disease-causing potential of SVs.
\end{abstract}

Technology-based approaches for the quantification of chromatin contacts have shown that mammalian genomes are folded in a highly controlled manner and that the resulting 3D-configuration directly influences gene regulation ${ }^{1}$. Hi-C, a genome-wide variant of chromosome conformation capture (3C) technologies ${ }^{2}$, has shown that mammalian genomes are organized in topologically associating domains (TADs), large genomic regions that display a high degree of interaction and that are largely conserved across species, cell types, and tissue types ${ }^{3-6}$. TADs are separated by boundaries that constrain interactions between enhancers and their target genes. The $3 \mathrm{D}$ folding of the genome and especially the organization of TADs can be disrupted by genomic rearrangements, such as deletions, duplications, or inversions, collectively called structural variants $(\mathrm{SVs})^{7-10}$. SVs can result in a rewiring of enhancer-promoter contacts, gene misexpression, and disease. However, it is currently difficult to predict such ectopic interactions without performing extensive $3 \mathrm{C}$ studies in cells or tissues carrying the rearranged chromosomes.

Polymer models have been successfully employed to dissect the $3 \mathrm{D}$ organization of chromosomes. In one strategy, chromatin is described as beads on a chain, and their folding is fitted to represent an average $3 \mathrm{D}$ structure that matches $\mathrm{Hi}-\mathrm{C}$ interaction frequencies ${ }^{11-13}$.
3D chromatin conformations can also be derived using models from polymer physics and previous knowledge of chromatin factors, notably CTCF and other transcription factors ${ }^{14-22}$. Here we focus on the strings and binders switch (SBS) polymer model ${ }^{19}$, which has been previously shown to recapitulate $\mathrm{Hi}-\mathrm{C}$ and FISH data to a high degree $^{14,17}$. The SBS model considers the interactions between chromatin filaments and cognate molecular binders. A chromatin filament is represented as a self-avoiding string of beads (Fig. 1a). The string contains inert (gray) beads, which do not interact with other factors apart from steric hindrance, and bridging beads, which are binding sites for cognate binding molecules that can form loops.

Based on the SBS polymer model, we developed a simulated annealing Monte Carlo optimization procedure, named PRISMR (polymer-based recursive statistical inference method), to infer the minimal factors that shape chromatin folding and its equilibrium 3D structure under the laws of physics, without a priori assumptions and with no additional or tunable parameters. PRISMR scans through the space of all polymer models to find the minimum of a cost function that takes into account the distance between the input $\mathrm{Hi}-\mathrm{C}$ matrix and the analogous contact matrix derived by polymer thermodynamics for the given model, and an additional Bayesian term to reduce overfitting by penalizing overestimation of binding site number and type (see Methods and Supplementary Fig. 1). The output of PRISMR at the cost-function convergence is the optimal polymer model of the genomic region of interest, with the minimum number and type of required binding sites, to reproduce the experimental Hi-C matrix (Fig. $1 \mathrm{~b}$ and see Methods). Although PRISMR models are derived from $\mathrm{Hi}-\mathrm{C}$ pairwise contacts, they can be used to derive any further aspect of folding (such as the ensemble of $3 \mathrm{D}$ conformations that the given locus assumes, its higher-order contacts, or the physical distances of genes and regulatory regions) and to predict the effect of rearrangements (Fig. 1c).

To test the predictive value of PRISMR, we chose the EPHA4 locus, a key developmental region associated with different types of limb malformations ${ }^{7}$. In a previous study, we showed by circular chromosome conformation capture sequencing (4C-seq) that deletions, inversions, and duplications cause distinct phenotypes (brachydactyly, syndactyly, polydactyly) by altering the chromatin organization of the locus, thereby causing rewiring of enhancer-promoter contacts and gene misexpression ${ }^{7}$. To study the

'Dipartimento di Fisica, Università di Napoli Federico II, and INFN Napoli, Complesso di Monte Sant'Angelo, Naples, Italy. ${ }^{2}$ Max Planck Institute for Molecular Genetics, RG Development and Disease, Berlin, Germany. ${ }^{3}$ Institute for Medical and Human Genetics, Charité - Universitätsmedizin Berlin, Berlin, Germany. ${ }^{4}$ Berlin-Brandenburg Center for Regenerative Therapies (BCRT), Charité - Universitätsmedizin Berlin, Berlin, Germany. ${ }^{5}$ Department of Computational Molecular Biology, Max Planck Institute for Molecular Genetics, Berlin, Germany. ${ }^{6}$ Department Developmental Genetics, Max Planck Institute for Molecular Genetics, Berlin, Germany. ${ }^{7}$ Epigenetic Regulation and Chromatin Architecture Group, Berlin Institute for Medical Systems Biology, Max-Delbrück Center for Molecular Medicine, Berlin-Buch, Germany. ${ }^{8}$ Present address: Epigenetics and Sex Development Group, Berlin Institute for Medical Systems Biology, Max-Delbrück Center for Molecular Medicine, Berlin-Buch, Germany. ${ }^{9}$ These authors contributed equally: Simona Bianco, Darío G. Lupiáñez, Andrea M. Chiariello, Carlo Annunziatella. *e-mail: mundlos@molgen.mpg.de; mario.nicodemi@na.infn.it 


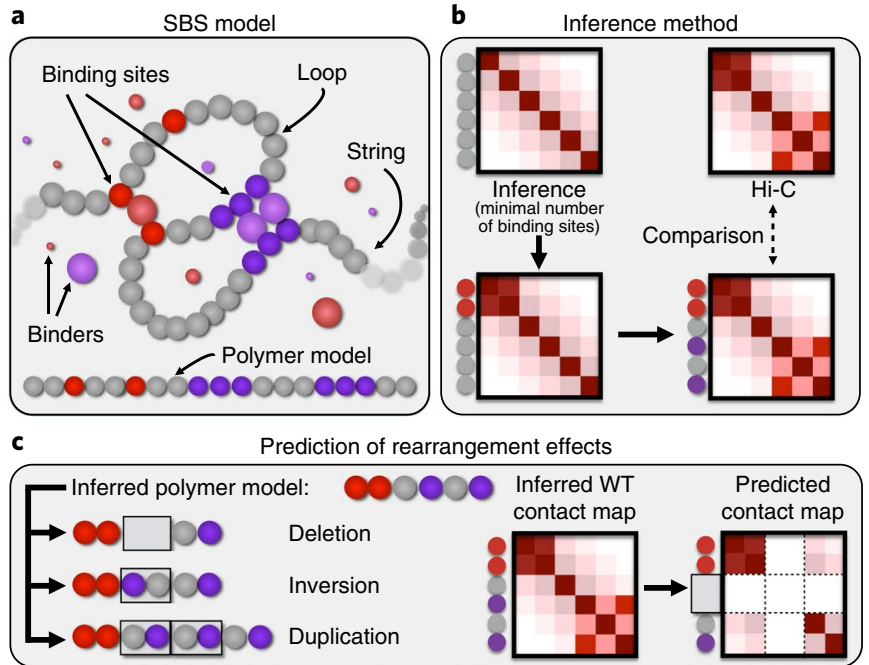

Fig. 1 | The PRISMR method: inference of molecular binders shaping chromatin folding. a, In the SBS model, chromatin is represented as a chain of beads interacting with molecular binders, in which the different types of binding sites (and their cognate bridging molecules) are visualized in different colors. b, PRISMR samples the thermodynamics ensemble of the conformations of a given SBS model to derive its contact matrix from polymer physics. By a simulated annealing Monte Carlo algorithm (SA), it iteratively finds the model that best describes the input contact matrix. c, By informing the model inferred from wild-type (WT) data with a given rearrangement, the effects of genomic mutations on folding can be predicted from only polymer physics without any fitting parameters.

$3 \mathrm{D}$ configuration of the entire locus, we performed capture $\mathrm{Hi}-\mathrm{C}$ $(\mathrm{cHi}-\mathrm{C})^{10}$ on embryonic day (E) 11.5 mouse limb buds and human skin fibroblasts. We also analyzed previously published Hi-C datasets from murine CH12-LX and human IMR90 cells $^{23}$. Regardless of the cell or tissue type or the species, we observed a subdivision of the locus in one large TAD, containing only EPHA4, in a smaller TAD, containing $P A X 3$ and $S G P P 2$, and in a gene-dense region on the centromeric side, showing no clear TAD structure (Fig. 2a). Differences were apparent within the EPHA4 TAD that likely reflect cell- and tissue-specific patterns of interaction and gene regulation ${ }^{5}$.

Toward developing predictive models of the architecture of the EPHA4 locus across different cell types, we applied PRISMR to all four $\mathrm{Hi}-\mathrm{C}$ datasets. The derived contact matrices were similar to the original Hi-C data, not only recapitulating the global TAD conformation of the locus, but also capturing cell-specific intra-TAD organization (Fig. 2a and Supplementary Note): the Pearson correlation, $r$, and distance-corrected correlation coefficient, $r^{\prime}$, range up to $r=0.95$ and $r^{\prime}=0.69$ (Fig. 2a, Methods, Supplementary Fig. 2, and Supplementary Table 1). PRISMR identifies the different binding domains of the locus, i.e., the sets of binding sites of the same type (i.e., color) that determine the folding patterns (Fig. 2b). In CH12-LX cells, for instance, the model predicted 21 different binding domains. They are all required to meet the accuracy cutoff in the description of Hi-C data, and six of them had main structural roles (see Methods). To check the robustness of our simulated annealing Monte Carlo procedure, we investigated whether there were differences between the best minima achieved by different runs of the PRIMSR simulations. We found that independent runs gave correlations comparable with those of the experimental $\mathrm{Hi}-\mathrm{C}$ data and similar corresponding polymer models $\left(P<1 \times 10^{-250}\right.$; Supplementary Note); additionally, the optimal model was robust to changes of the number of binding domain types (Supplementary Note). The model binding domains can be broadly divided into two categories (Fig. $2 \mathrm{~b}$ and see Methods): one category includes domains largely overlapping with annotated TADs (type-I), and the other includes domains extending over several TADs or even over the whole analyzed region (type-II). Comparing the genomic position of the model binding sites with ENCODE epigenetics data available for CH12-LX cells ${ }^{24}$ showed that a single binding type (a 'color') did not correlate with a single molecular factor (see Methods and Supplementary Fig. 3).

To explore the roles of various factors to the folding patterns detected by PRISMR simulations of $\mathrm{cHi}-\mathrm{C}$ data, we considered the architectural protein CTCF, a DNA-binding transcription factor thought to facilitate the formation of chromatin loops ${ }^{20,25,26}$. Notably, some binding site types identified by PRISMR correlated with CTCF (Supplementary Fig. 3). As PRISMR does not exploit prior information on binding sites and factors, to test its reach we considered a variant of the model in which we included previous knowledge about the location of CTCF binding sites in the locus, which were added to interact with an additional type of binder that bridges opposed (forward or reverse) CTCF sites (see Methods). In limb tissue E11.5 cells, for instance, this variant (named 'PRISMR + CTCF') had correlations with $\mathrm{Hi}-\mathrm{C}$ data similar to those of the initial model: it improved the visualization of the large Epha4 TAD, mainly by strengthening the loop anchors characteristic for CTCF-associated loops, but it also resulted in additional contacts in the neighboring gene-dense region that were not present in the original $\mathrm{cHi}-\mathrm{C}$ data (Fig. 2c and Supplementary Fig. 4). Conversely, a model with only CTCF (named 'CTCF-only') can describe some of the loops seen in the data ${ }^{20}$, but poorly captured the global contact patterns of the Epha4 locus (Supplementary Fig. 4), resulting in a lower correlation coefficient $\left(r^{\prime}=0.05\right)$. These results indicate that other factors besides CTCF were important in chromatin folding and TAD configuration (see Methods) and that our approach can recapitulate most of the interactions of $\mathrm{Hi}-\mathrm{C}$ data without a priori information on binding factors. Nevertheless, such information can be added to adapt and improve model predictions.

To test whether PRISMR can predict the effects of homozygous SVs on chromatin folding, we investigated three previously reported variants ${ }^{7}$ at the Epha4 mouse locus: a deletion (DelB) encompassing a large part of the Epha4 TAD and the telomeric TAD boundary (associated with brachydactyly due to misexpression of Pax3), a slightly smaller deletion (DelBs) that leaves the TAD boundary intact (no misexpression, no phenotype), and a balanced $1.1-\mathrm{Mb}$ inversion $(\operatorname{InvF})$ that causes misexpression of Wnt6. We implemented these mutations in polymer models of the wild-type CH12-LX and E11.5 limbs cells inferred by PRISMR and re-ran the ensemble of folding conformations to derive an average locus contact matrix. For E11.5 limb tissue, we tested both the PRISMR model and the PRISMR + CTCF version with the addition of CTCF sites (Fig. 3a and Supplementary Figs. 5a, 6a, and 7). To identify the regions of statistically significant ectopic interactions in each predicted rearrangement, we subtracted each mutant matrix from the wild-type matrix (Fig. 3b and Supplementary Figs. 5b, 6b, and 8). Although the studied locus is populated by more than 40 genes, our matrices predicted that only certain regions, containing a limited number of genes, would display changes in the interaction profiles. For example, in the larger deletion (DelB) including the Epha4 TAD boundary, we identified new contacts that predicted fusion between the remaining Epha4 and Pax3 TADs, thus facilitating the association between Epha4 enhancers and Pax3 that results in ectopic gene activation and a pathogenic phenotype $\mathrm{e}^{7}$. Ectopic contacts between the same regions were also predicted in the smaller deletion (DelBs), which leaves the Epha4-Pax3 boundary intact. However, virtual $4 \mathrm{C}$ analysis derived from our predictions showed that the enhancers-Pax3 ectopic interaction was diminished, consistent with the absence of Pax3 activation in these mutants (Fig. $3 \mathrm{c}$ and Supplementary Figs. $5 c$ and $6 c)$. The inversion $(\operatorname{InvF})$ was predicted to result in a rearrangement of the genomic content of the two 
a

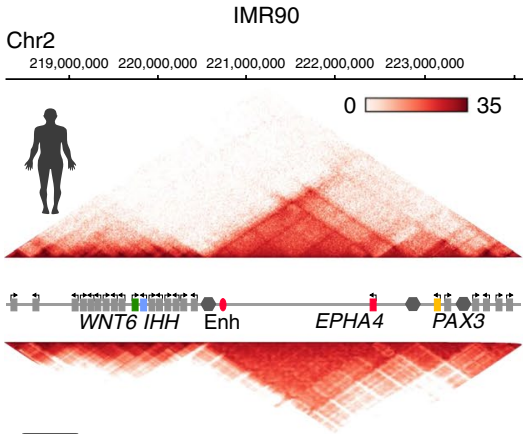

PRISMR

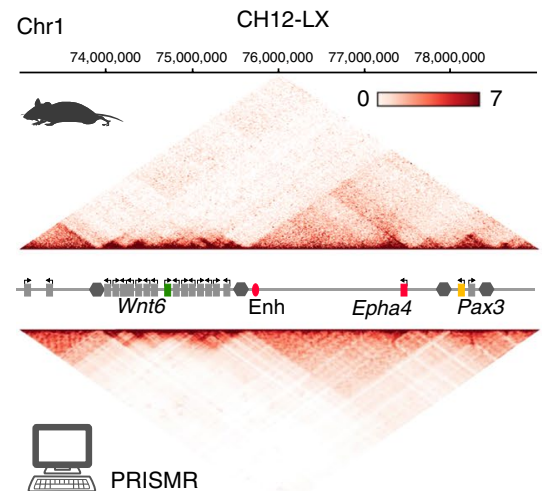

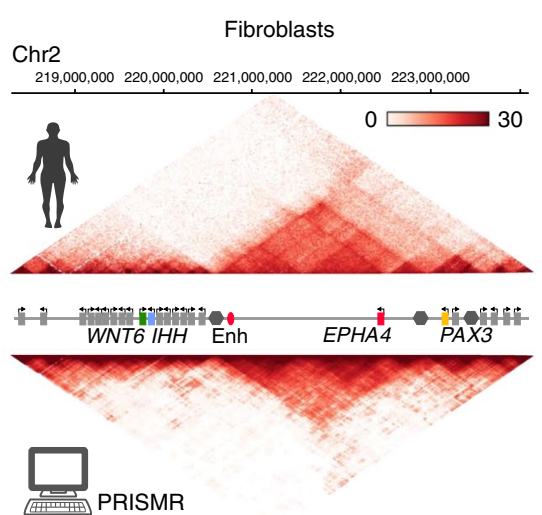

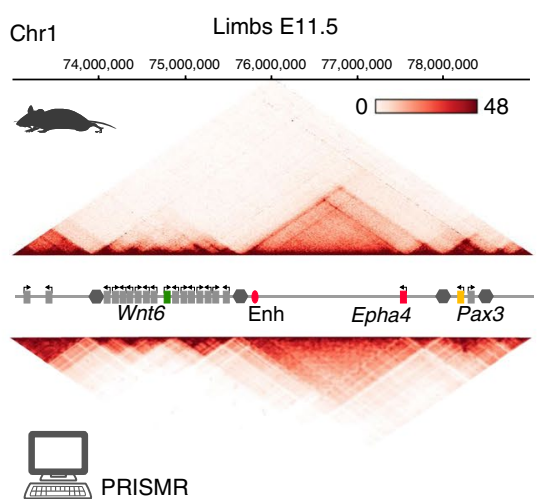

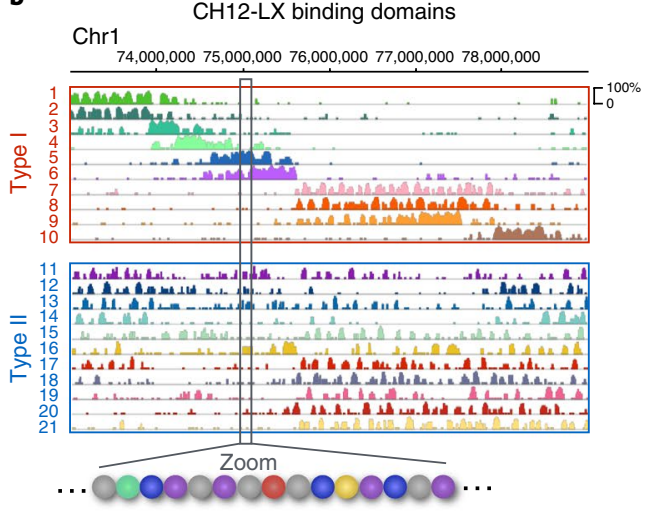

c

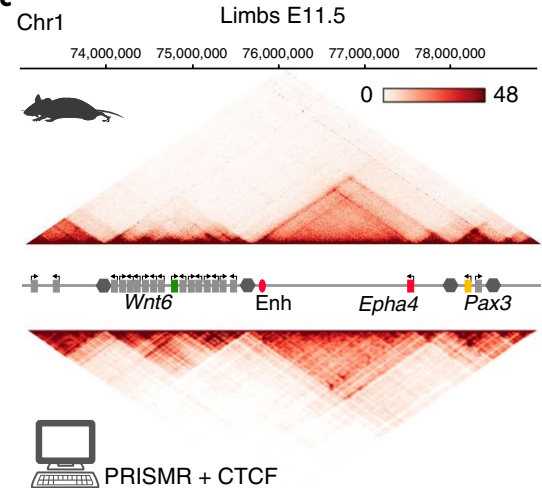

Fig. 2 | PRISMR recapitulates 3D conformation at the EPHA4 locus. a, Published $\mathrm{Hi}-\mathrm{C}$ data ${ }^{23}$ (left) and our capture $\mathrm{Hi}-\mathrm{C}$ data (right; $n=1$ with an internal control comparing 4 different experiments; see Methods) compare well with the contact matrices derived by PRISMR. Their Pearson correlations, $r$, and distance-corrected Pearson correlation coefficients, $r^{\prime}$, are comparatively high: $r=0.92, r^{\prime}=0.64$ in IMR90; $r=0.93, r^{\prime}=0.69$ in human fibroblasts; $r=0.91, r^{\prime}=0.56$ in $\mathrm{CH} 12-\mathrm{LX}$; and $r=0.94, r^{\prime}=0.60 \mathrm{in}$ limb tissue E11.5. The genomic region, with genes (rectangles), TAD boundaries (hexagons), and enhancers (ovals), is shown schematically. Relevant genomic elements are highlighted with colors and names. Enh, enhancer; chr, chromosome. b, PRISMR also identifies the different binding sites (see Fig. 1a) along the locus that shape its 3D structure. The binding sites of the same type (color) form the shown different binding domains. In $\mathrm{CH} 12-\mathrm{LX}$ cells, for example, there are 21 statistically significant (one-sided Wilcoxon's rank-sum test; $P<1.1 \times 10^{-7}$; see Supplementary Note) different binding domains. The plots represent their abundance (as percentages) along the genomic sequence. Type-I domains are spatially restricted, whereas type-II are ubiquitous, covering the whole region. Their overlapping genomic positions produce the observed complex interaction patterns. c, The contact matrix from a variant of the PRISMR model with addition of CTCF binding sites (PRISMR + CTCF model) has a similar Pearson correlation with experimental data ( $n=1$ with an internal control comparing 4 different experiments; see Methods); for example, in limb E11.5 tissue we find $r=0.95, r^{\prime}=0.52$.

adjacent TADs with interaction hotspots between Epha4 enhancers and a gene-dense region (three genes affected) that would be consistent with the ectopic Wnt6 activation reported previously. We also observed ectopic interactions between a region near the centromeric breakpoint containing the Wnt $10 a$ gene and the remaining Epha4 TAD. Therefore, PRISMR identified specific and localized regions of ectopic interactions across the entire locus as a consequence of genomic rearrangements, identifying a small number of genes whose regulation might be directly affected.

As a next step, we tested the accuracy of our predictions by comparison against a new experimental $\mathrm{cHi}-\mathrm{C}$ dataset from mouse limb buds carrying homozygous mutations. Our dataset showed the same regions of ectopic interaction and displayed a noticeably high agreement with PRISMR predictions, not only across the entire locus but also when the regions of ectopic interaction were compared (Fig. 3, Supplementary Tables 1 and 2, and Supplementary Figs. 5, 6, and 8). Our results confirmed that the larger deletion in DelB mutant led to a fusion of the Epha4 and Pax3 TADs, not occurring in the smaller DelBs mutation, in which the TAD boundary remains intact (Supplementary Fig. 9). In the inversion, ectopic contacts were observed between Wnt6 and the Epha4 enhancer region, which facilitated Wnt6 activation as previously observed in vivo $^{7}$, and between a region at the centromeric breakpoint and the entire Epha4 TAD. Notably, the observed ectopic interaction was interrupted by the Epha4 centromeric boundary, which, although inverted, appeared to retain its functionality (Supplementary Fig. 9). Hence, deletions and inversions that include boundary elements can result in fusions or reorganization of TADs, respectively.

Finally, we wanted to test the potential of PRISMR to predict the effects of heterozygous SVs on chromatin organization as they are commonly observed in human patient samples. A PRISMR polymer model of the EPHA4 locus, inferred from healthy control human fibroblast cHi-C data (Fig. 2a), was employed to predict the effects of SVs on chromatin contact matrices (Fig. 4 and Supplementary Fig. 10). To test the model predictions, we used fibroblasts obtained from human patients to perform cHi-C (Fig. 4). We analyzed a 1.6$\mathrm{Mb}$ deletion associated with brachydactyly (similar to mouse $\mathrm{DelB}$ ), a 900-kb duplication (DupP) associated with polydactyly and $I H H$ activation, and a 1.4-Mb duplication $(D u p F)$ associated with syndactyly and $W N T 6$ activation ${ }^{7}$. Subtraction maps identified the precise regions and intensity of significant ectopic interactions (Fig. 4b, Methods, and Supplementary Fig. 11). In the brachydactyly-associated deletion, 
a

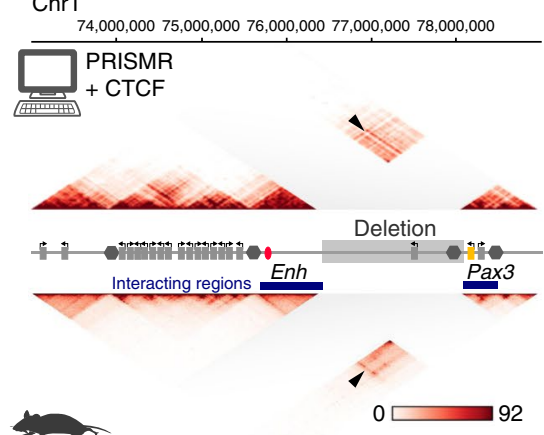

b Chr1

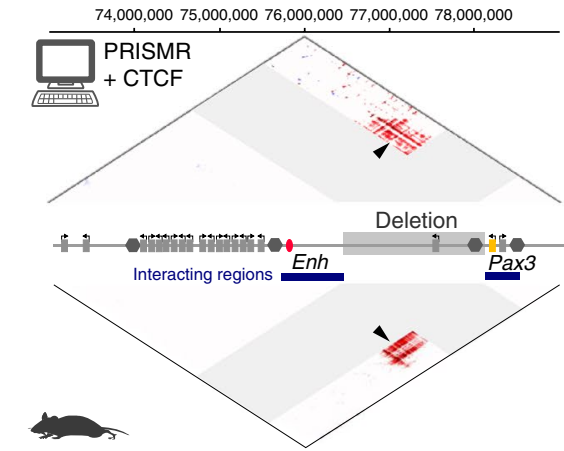

c $\mathrm{Chr} 1$

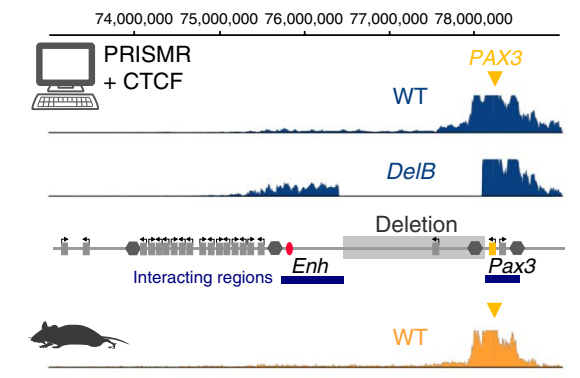

Chr1

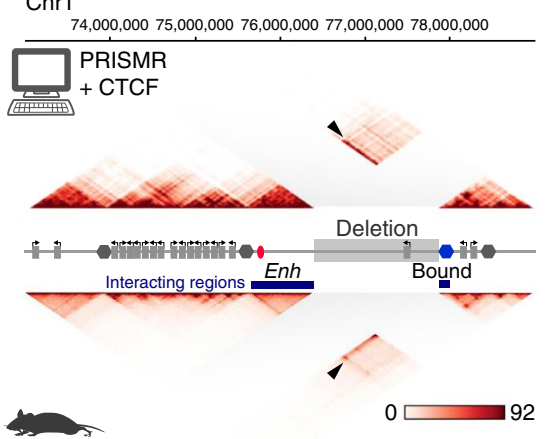

Chr1

$74,000,000$ 75,000,000 76,000,000 77,000,000 78,000,000
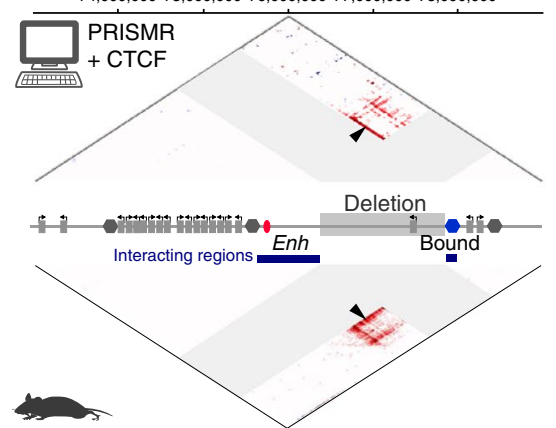

Chr1
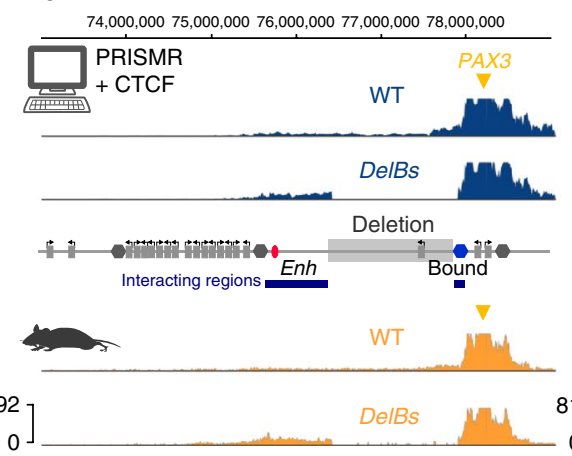
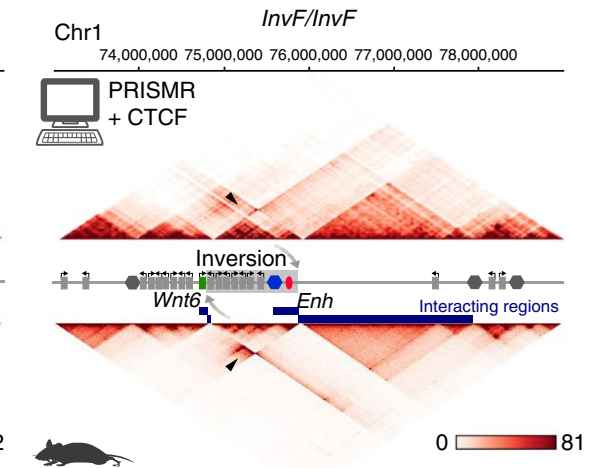

Chr1

$74,000,00075,000,000$ 76,000,000 77,000,000 78,000,000

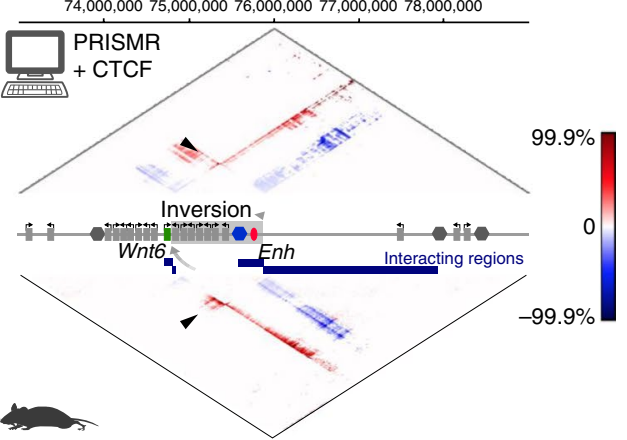

Chr1

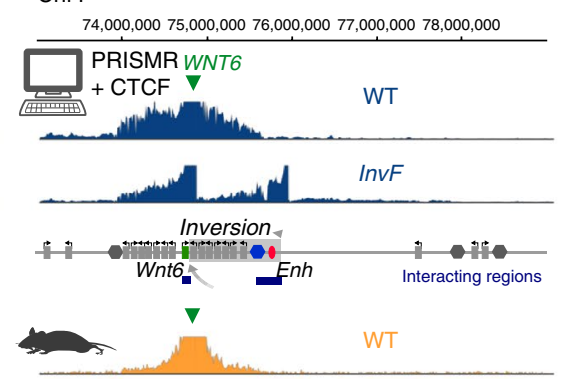

$\left.\begin{array}{c}81 \\ 0\end{array}\right]$

InvF

Fig. 3 | PRISMR predicts the effects of mouse homozygous structural variants on chromatin architecture. a, Contact matrices from model predictions derived from WT data (top) and cHi-C experiments performed in E11.5 limb buds from mouse mutants (bottom; $n=1$ with an internal control comparing 4 different experiments; see Methods). Schematic of genomic region displays genes (rectangles), TAD boundaries (hexagons), enhancers (ovals), and corresponding structural variant. DelB/DelB: PRISMR prediction on a 1.6-Mb homozygous deletion affecting Epha4 TAD and Epha4-Pax3 boundary (Pearson correlation $r=0.95$, distance-corrected Pearson correlation $r^{\prime}=0.41$ ). Note the increased interaction between remaining Epha4 and Pax3 TADs (arrowhead and blue bars). DelBs/DelBs: 1.5-Mb deletion affecting Epha4 TAD but not the Epha4-Pax3 boundary $\left(r=0.95, r^{\prime}=0.50\right)$. Note the increased interaction between remaining Epha4 TAD and Epha4-Pax3 boundary (blue hexagon). InvF/InvF: 1.1-Mb homozygous inversion $\left(r=0.95, r^{\prime}=0.60\right)$. Note the increased interaction between enhancer and Wnt6 regions. An additional region at the centromeric inverted position (containing Wnt10a) gains interaction with Epha4 TAD. The centromeric Epha4 boundary retains functionality despite inversion (blue hexagon). b. Subtraction maps (WT and mutants) from predictions and $\mathrm{cHi}-\mathrm{C}$ data ( $n=1$ with an internal control comparing 4 different experiments; see Methods). Top: threshold gain (red) and loss (blue) of interaction is displayed (absolute differences $>2$ s.d.; see Methods). Ectopic interactions are indicated (arrowheads and blue bars). c, Virtual $4 \mathrm{C}$ plots derived from predictions and $\mathrm{cHi}-\mathrm{C}$ data from viewpoints on the respective phenotype-causing genes. DelB/DelB: note increased interaction of the Pax3 promoter with the remaining Epha4 TAD, including enhancer cluster in both prediction and experimental data. DelBs/DelBs: the Pax3 promoter interacts less frequently with Epha4 TAD compared to DelB/DelB mutants. InvF/InvF: increased interaction between Wnt6 gene and Epha4 enhancer cluster.

both PRISMR and the fibroblast-derived $\mathrm{cHi}-\mathrm{C}$ data detected the same region of ectopic interaction as seen in the equivalent mouse mutant DelB, displaying increased interaction between $P A X 3$ and the EPHA4 enhancer cluster. In the duplication $D u p F$, we observed ectopic interactions not only between the enhancer cluster and WNT6, but also with the neighboring gene WNT10A. In DupP, the diseasecausing gene $I H H$ displayed increased interaction with the enhancers, as well as with the two neighboring genes, CCDC108 and NHEJ1.
Comparison of the PRISMR predictions with cHi-C data from patient fibroblasts revealed a high correlation (Supplementary Tables 1 and 2), demonstrating that PRISMR can also predict the effects of SVs on misfolded chromatin contacts in heterozygous samples, thus facilitating the identification of disease-causing genes.

Collectively, our results demonstrate that PRISMR is an efficient tool for predicting alterations in chromatin contacts induced by disease-associated SVs in both homozygous and heterozygous samples 
Chr2 DelB/t+

$219,000,000 \quad 220,000,000221,000,000 \quad 222,000,000223,000,000$
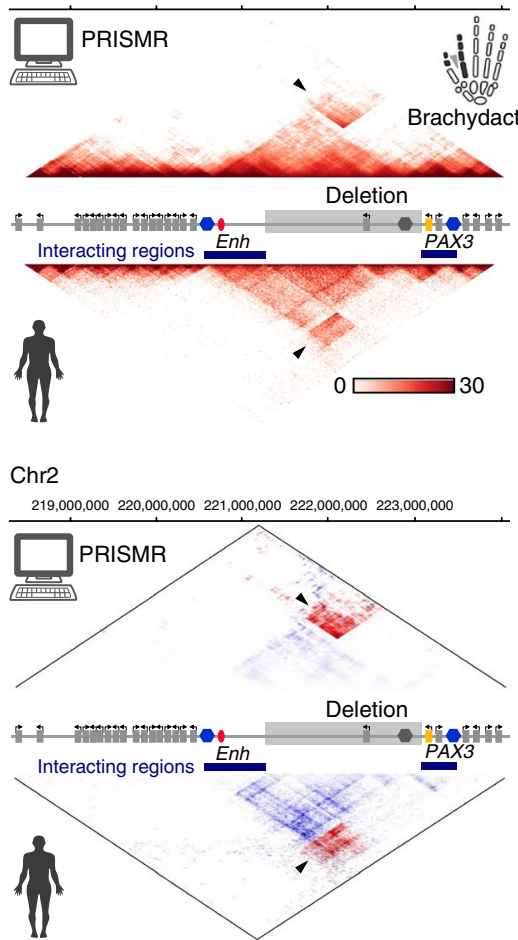

C Chr2
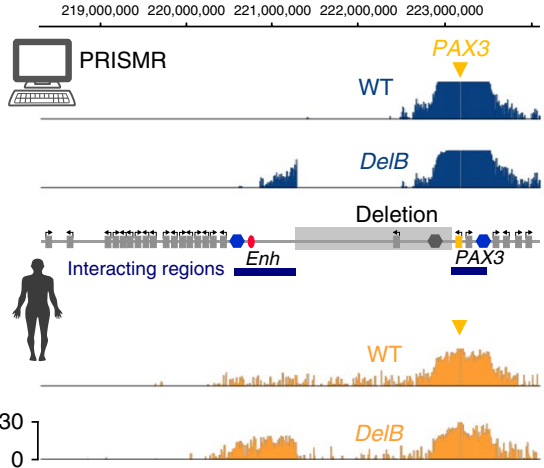

Chr2

DupF/+

$219,000,000220,000,000221,000,000222,000,000223,000,000$
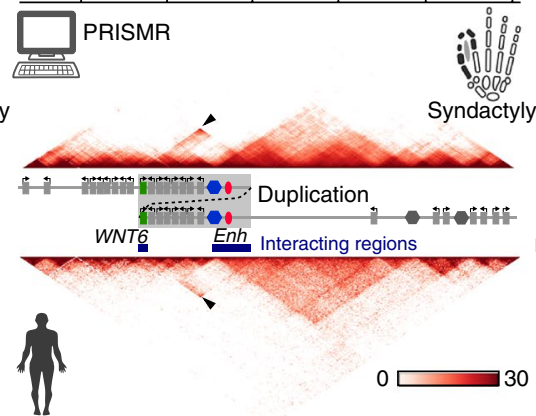

Chr2

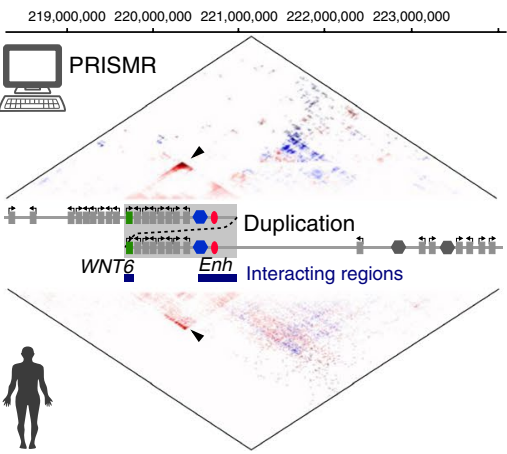

Chr2

$219,000,000220,000,000221,000,000222,000,000223,000,000$
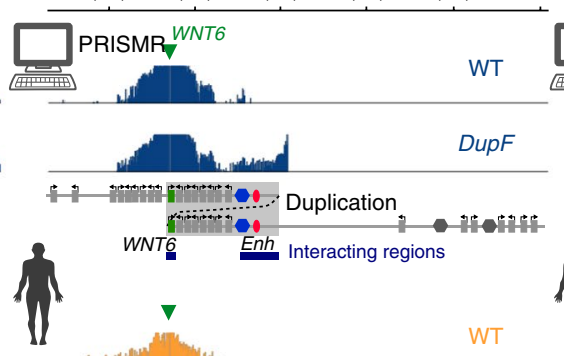

$\left.\begin{array}{r}30 \\ 0\end{array}\right]$
Chr2

DupP/+

$219,000,000220,000,000221,000,000222,000,000223,000,000$
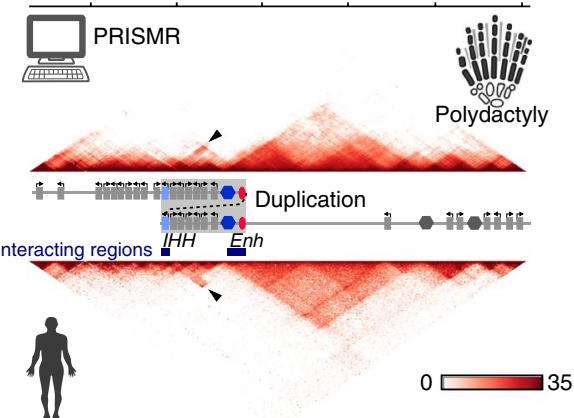

Chr2

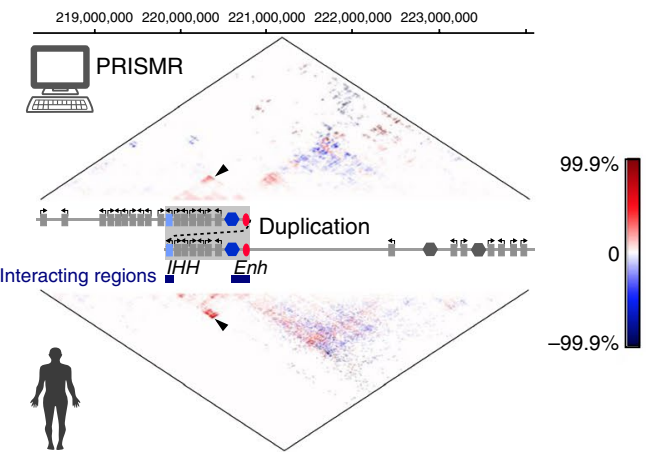

Chr2

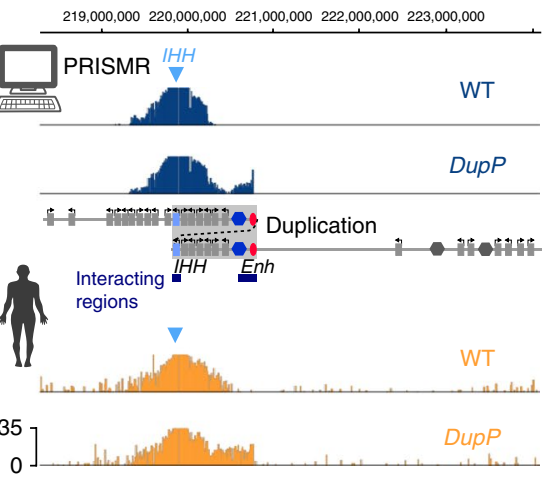

Fig. 4 | PRISMR predicts the effects of human heterozygous structural variants on chromatin architecture. a, Contact matrices from model predictions derived from WT data (top) and cHi-C experiments in mutation carrying cultured human skin fibroblasts (bottom; $n=1$ with an internal control comparing 4 different experiments; see Methods). Schematic genomic region displays genes (rectangles), TAD boundaries (hexagons), enhancers (ovals), and corresponding structural variant. Human phenotypes associated with the rearrangement are indicated on right. DeIB/ + : PRISMR predicts the chromatin effects of a 1.6-Mb heterozygous deletion (Pearson correlation $r=0.93$, distance-corrected Pearson correlation $r^{\prime}=0.61$ ). Increased interaction is detected between the remaining EPHA4 and PAX3 TADs (arrowhead and blue bars), resulting in PAX3 misexpression and brachydactyly. DupF/ + : heterozygous $1.4-\mathrm{Mb}$ duplication $\left(r=0.88, r^{\prime}=0.52\right)$. Increased interaction is detected between EPHA4 enhancer cluster and WNT6 regions. DupP/ + : heterozygous 900-bp duplication $\left(r=0.90, r^{\prime}=0.56\right)$. Increased interaction is detected between EPHA4 enhancer cluster and $\mathrm{IHH}$ regions. b, Subtraction maps produced (using a healthy control and patients) from predictions and cHi- $\mathrm{C}$ data ( $n=1$ with an internal control comparing 4 different experiments; see Methods). Above, threshold gain of interaction is displayed in red and loss in blue (absolute differences > 2 s.d.; see Methods). Ectopic interactions between EPHA4 TAD and genomic regions are indicated (arrowheads and blue bars). c, Virtual $4 \mathrm{C}$ plots derived from predictions and $\mathrm{cHi}-\mathrm{C}$ data from the viewpoint on the respective phenotype-causing gene. DelB $/+$ : note increased interaction of PAX3 promoter with remaining EPHA4 TAD, including EPHA4 enhancer cluster in both, prediction and experimental data. DupF/ + : note increased interaction of WNT6 promoter with the EPHA4 enhancer cluster. DupP/ + : note increased interaction of IHH promoter with the EPHA4 enhancer cluster.

and even in complex genomic regions with high gene density. PRISMR predictions can be used to identify regions of ectopic interaction that can then be scanned for their content, i.e., the presence of genes and enhancers that could interact. Furthermore, our results indicate that PRISMR can be used in cases where affected tissues or equivalent cell types are not available. Recent advances in high-throughput sequencing have boosted the identification of $\mathrm{SVs}^{27-29}$. In this scenario, polymer modeling by PRISMR emerges as a valid approach for predicting pathogenic effects, facilitating the interpretation and diagnosis of this type of genomic rearrangement. 


\section{Methods}

Methods, including statements of data availability and any associated accession codes and references, are available at https://doi. org/10.1038/s41588-018-0098-8.

Received: 4 January 2017; Accepted: 27 February 2018; Published online: 16 April 2018

\section{References}

1. Fraser, J., Williamson, I., Bickmore, W. A. \& Dostie, J. An overview of genome organization and how we got there: from FISH to Hi-C. Microbiol. Mol. Biol. Rev. 79, 347-372 (2015).

2. Lieberman-Aiden, E. et al. Comprehensive mapping of long-range interactions reveals folding principles of the human genome. Science 326, 289-293 (2009).

3. Dixon, J. R. et al. Topological domains in mammalian genomes identified by analysis of chromatin interactions. Nature 485, 376-380 (2012).

4. Nora, E. P. et al. Spatial partitioning of the regulatory landscape of the X-inactivation centre. Nature 485, 381-385 (2012).

5. Phillips-Cremins, J. E. et al. Architectural protein subclasses shape 3D organization of genomes during lineage commitment. Cell 153, 1281-1295 (2013)

6. Fraser, J. et al. Hierarchical folding and reorganization of chromosomes are linked to transcriptional changes in cellular differentiation. Mol. Syst. Biol. 11 852 (2015).

7. Lupiáñez, D. G. et al. Disruptions of topological chromatin domains cause pathogenic rewiring of gene-enhancer interactions. Cell 161, 1012-1025 (2015).

8. Hnisz, D. et al. Activation of proto-oncogenes by disruption of chromosome neighborhoods. Science 351, 1454-1458 (2016).

9. Lupiáñez, D. G., Spielmann, M. \& Mundlos, S. Breaking TADs: how alterations of chromatin domains result in disease. Trends Genet. 32, 225-237 (2016).

10. Franke, M. et al. Formation of new chromatin domains determines pathogenicity of genomic duplications. Nature 538, 265-269 (2016).

11. Duan, Z. et al. A three-dimensional model of the yeast genome. Nature 465 , 363-367 (2010).

12. Lesne, A., Riposo, J., Roger, P., Cournac, A. \& Mozziconacci, J. 3D genome reconstruction from chromosomal contacts. Nat. Methods 11 , 1141-1143 (2014).

13. Serra, F et al. Restraint-based three-dimensional modeling of genomes and genomic domains. FEBS Lett. 589(20 Pt A), 2987-2995 (2015).

14. Barbieri, M. et al. Complexity of chromatin folding is captured by the strings and binders switch model. Proc. Natl. Acad. Sci. USA 109, 16173-16178 (2012).

15. Bohn, M. \& Heermann, D. W. Diffusion-driven looping provides a consistent framework for chromatin organization. PLoS One 5, e12218 (2010).

16. Brackley, C. A., Taylor, S., Papantonis, A., Cook, P. R. \& Marenduzzo, D. Nonspecific bridging-induced attraction drives clustering of DNA-binding proteins and genome organization. Proc. Natl. Acad. Sci. USA 110, E3605-E3611 (2013).

17. Chiariello, A. M., Annunziatella, C., Bianco, S., Esposito, A. \& Nicodemi, M. Polymer physics of chromosome large-scale 3D organisation. Sci. Rep. 6, 29775 (2016).

18. Giorgetti, L. et al. Predictive polymer modeling reveals coupled fluctuations in chromosome conformation and transcription. Cell 157, 950-963 (2014).
19. Nicodemi, M. \& Prisco, A. Thermodynamic pathways to genome spatial organization in the cell nucleus. Biophys. J. 96, 2168-2177 (2009).

20. Sanborn, A. L. et al. Chromatin extrusion explains key features of loop and domain formation in wild-type and engineered genomes. Proc. Natl. Acad. Sci. USA 112, E6456-E6465 (2015).

21. Scialdone, A., Cataudella, I., Barbieri, M., Prisco, A. \& Nicodemi, M. Conformation regulation of the $\mathrm{X}$ chromosome inactivation center: a model. PLoS Comput. Biol. 7, e1002229 (2011).

22. Fudenberg, G. et al. Formation of chromosomal domains by loop extrusion. Cell Rep. 15, 2038-2049 (2016).

23. Rao, S. S. et al. A 3D map of the human genome at kilobase resolution reveals principles of chromatin looping. Cell 159, 1665-1680 (2014).

24. ENCODE Project Consortium. An integrated encyclopedia of DNA elements in the human genome. Nature 489, 57-74 (2012).

25. Guo, Y. et al. CRISPR inversion of CTCF sites alters genome topology and enhancer/promoter function. Cell 162, 900-910 (2015).

26. Nora, E. P. et al. Targeted degradation of CTCF decouples local insulation of chromosome domains from genomic compartmentalization. Cell 169, 930-944.e22 (2017).

27. Gilissen, C. et al. Genome sequencing identifies major causes of severe intellectual disability. Nature 511, 344-347 (2014).

28. Hehir-Kwa, J. Y. et al. A high-quality human reference panel reveals the complexity and distribution of genomic structural variants. Nat. Commun. 7, 12989 (2016).

29. Newman, S., Hermetz, K. E., Weckselblatt, B. \& Rudd, M. K. Next-generation sequencing of duplication CNVs reveals that most are tandem and some create fusion genes at breakpoints. Am. J. Hum. Genet. 96, 208-220 (2015).

\section{Acknowledgements}

We thank the sequencing core, transgenic unit, and animal facilities of the Max Planck Institute for Molecular Genetics for technical assistance. This work is supported by grants from the Deutsche Forschungsgemeinschaft (DFG) and the Max Planck Foundation (MPF) to S.M. and D.G.L., the Berlin Institute of Health (BIH) to S.M. and A.P.; by CINECA ISCRA Grant HP10CYFPS5 and HP10CRTY8P, computer resources at INFN and Scope at the University of Naples (M.N.), and the Einstein BIH Fellowship Award to M.N.

\section{Author contributions}

M.N. and S.M. designed the project. S.M., D.G.L., and M.V. devised the cHi-C experiments. S.B., A.M.C., C.A., and M.N. developed the modeling part; S.B., A.M.C., and C.A. ran the computer simulations and performed their analyses. L.W. derived mouse homozygous lines and performed tetraploid aggregations. D.G.L., K.K., and G.A performed cHi-C experiments, and R.S. performed bioinformatic analyses. M.N., S.M., D.G.L., S.B., A.P., A.M.C., and C.A. wrote the manuscript.

\section{Competing interests}

The authors declare no competing interests.

\section{Additional information}

Supplementary information is available for this paper at https://doi.org/10.1038/ s41588-018-0098-8.

Reprints and permissions information is available at www.nature.com/reprints. Correspondence and requests for materials should be addressed to S.M. or M.N.

Publisher's note: Springer Nature remains neutral with regard to jurisdictional claims in published maps and institutional affiliations. 


\section{Methods}

The studied loci. The murine Epha4 locus discussed in this paper is a 6-Mb long region around the Epha4 gene (coordinates mm9 chr1: 73,000,000-79,000,000). We employed in situ Hi-C data from CH12-LX cells ${ }^{23}$ and our own cHi-C data in E11.5 mouse limb buds, at a 10-kb resolution. The studied human EPHA4 locus in skin fibroblasts is $5.77 \mathrm{Mb}$ long (coordinates hg19 chr2: 218,320,000-224,090,000); in that system we produced our own cHi-C data at $10-\mathrm{kb}$ resolution. We also studied the EPHA4 locus in human IMR90 cells, where we used previously published in situ Hi-C data at $10-\mathrm{kb}$ resolution ${ }^{23}$; the considered locus is $8 \mathrm{Mb}$ long (coordinates hg19 chr2: 217,000,000-225,000,000).

The strings and binders switch (SBS) model. The string and binders switch (SBS) polymer model of chromatin folding quantifies the biological scenario where molecules, such as transcription factors, loop DNA by bridging distal cognate binding sites ${ }^{14,19}$. In the SBS model, a chromatin filament is represented as a selfavoiding (SAW) polymer chain of $N$ beads. The beads can be bound and bridged by molecular binders; each type of bead only by its specific, cognate type of binders (Fig. 1a). In our notation, $n$ is the total number of different types of binding sites (represented with different colors in the figures). There are also inert sites along the chain, i.e., beads (represented in gray) that do not interact with any binder apart from steric hindrance. Each type of binder has a molar concentration $c$ and their binding energy to their cognate beads is $E_{\text {int }}$. As well described in polymer physics and in previous studies ${ }^{14,17,19}$, the above model exhibits a coil-globule phase transition, from an open conformation in the SAW universality class found at low $c$ or $E_{\text {int }}$ to a different conformational class corresponding to a globule, compact state $^{30}$. All details on the model and its simulations are given below and in the Supplementary Note.

The PRISMR algorithm. Our PRISMR algorithm aims to find the minimal number and types (colors) of binding sites in a SBS polymer chain, and their position along the chain, that best reproduces an input contact matrix of a given chromosomal locus, by folding solely according to the laws of physics (Fig. 1b). PRISMR is based on a standard simulated annealing Monte Carlo (SA) optimization procedure ${ }^{31,32}$ that minimizes the distance between the predicted polymer model and the input contact matrix, under a Bayesian weighting factor to avoid overfitting. The procedure involves five main iterative steps: (i) consider a polymer model with a given arrangement of binding sites; (ii) derive a thermodynamics ensemble of its 3D equilibrium conformations; (iii) compute its contact matrix; (iv) compare it with the input Hi-C data; and (v) change the polymer model accordingly and repeat until convergence. Those steps are described in full details below and in the Supplementary Note.

The sequence of the genomic region to be modeled is divided into $L$ windows according to the genomic resolution of the considered Hi-C experiment. As a single DNA window could include many binding sites, we consider a polymer chain that is $r$ times longer in order to include such details. The optimal value of $r$ is returned as an intermediate output by PRISMR, as described below. An SBS polymer model is identified by the arrangement of binding sites of different types along the chain of beads, i.e., by the set $\left\{c_{i}\right\}$ of its color variables $c_{i}=0,1, \ldots, n$ ( 0 corresponding to gray, inert sites), where the index $i=1, \ldots, N$ labels the $i$ th bead. The output of PRISMR is then the best, minimal arrangement $\left\{c_{i}\right\}_{m}$ of beads along the chain to describe the input contact matrix.

Specifically, the iterative SA procedure of the algorithm minimizes a cost function, $H$, including two terms to fit well the data and to avoid overfitting. The first one, $H_{0}$, is the distance between the input, $C_{\text {exp }}(i, j)$, and model, $C(i, j)$, contact matrices; the second, $H_{\lambda}$, is a Bayesian term (a chemical potential in statistical mechanics) that penalizes the addition of binding sites (see Supplementary Note for details). The weight of the Bayesian term is given by a positive factor $\lambda$ : the larger $\lambda$ is, the more the addition of binding sites is penalized (if $\lambda=0$, there is no penalty).

Given $n, r$, and $\lambda$, PRISMR samples the huge space of the possible arrangements, $\left\{c_{i}\right\}$, of the binding sites along the polymer chain (having $(n+1)^{N}$ elements) to search for the minimum, $\left\{c_{i}\right\}_{m}$, of the cost function $H$ by use of a standard SA iterative procedure ${ }^{31,32}$. Starting from a random initial assignment of the binding sites along the chain, the color of a randomly chosen bead is changed at random, the average contact matrix of the new polymer is computed, and the cost function evaluated until convergence (see Supplementary Note). The SA procedure is repeated, from many initial conditions, with different values of $n, r$, and $\lambda$ to find the minimal required number of different colors $n^{*}$, the minimal required value of $r, r^{\star}$, and the minimal number of binding sites, i.e., the maximum allowed value of $\lambda, \lambda^{*}$, to explain the input data within a given accuracy (Supplementary Fig. 1 and Supplementary Note). Since $r^{*}$ is systematically found to be smaller than $n^{*}$ in all the cases studied here, for simplicity we consider the safe option where $r^{*}=n^{*}$. The optimal arrangement of binding sites, $\left\{c_{i}\right\}^{*}$, obtained with $n^{*}$ and $\lambda^{*}$, is the final output of the PRISMR algorithm (as shown in Fig. 2b, in log scale).

To derive our polymer models of the Epha4 locus, we applied the above procedure to four different input $\mathrm{Hi}-\mathrm{C}$ datasets, all binned at $10-\mathrm{kb}$ resolution and KR (Knights and Ruiz) normalized ${ }^{33}$. In the studied murine Epha4 locus, the algorithm returns $n^{*}=21$ and $\lambda^{*}=1.0$ in both the published in situ Hi-C data of CH12-LX cells ${ }^{23}$ and in our limb tissue cHi-C data. In the considered human
EPHA4 locus, PRISMR finds $n^{*}=16$ and $\lambda^{*}=1.0$ in the published in situ Hi-C data in human IMR90 cells $^{23}$, and $n^{*}=24$ and $\lambda^{*}=1.0$ in the cHi-C data in human fibroblast produced in this study. To simplify the notation, $n^{*}$ and $r^{*}$ are renamed $n$ and $r$ in the Results and Discussions sections of this paper. All details about the PRISMR algorithm, its SA procedure, robustness and convergence, can be found below and in the Supplementary Note.

A computationally demanding step of PRISMR is the iterative calculation of the equilibrium thermodynamics average contact frequency, $C(i, j)$, for the sites of a given polymer model, during each iteration of the SA procedure. That can be achieved, for instance, by molecular dynamics (MD) computer simulations; however, these may require huge computational efforts. To speed up the computation of $C(i, j)$, we implemented a mean-field approximation, an approach typical of statistical mechanics ${ }^{34}$ (see Supplementary Note). To test our approximation, we compare its results against full-scale $\mathrm{MD}$ simulations of the optimal model $\left\{c_{i}\right\}^{\star}$ found by the SA procedure. We find that the contact matrices obtained by MD have a Pearson correlation with those derived under the mean-field scheme ranging from $r=0.91$ to $r=0.95$ across the studied cases (Supplementary Note and Supplementary Fig. 12). Unless otherwise stated, we always report the full MD results throughout the paper.

Analyses of the model binding domains. We ran different successful tests of the statistical significance and robustness of the optimal polymer models, $\left\{c_{i}\right\}^{*}$ identified by PRISMR (Supplementary Note). In particular, analyses were performed to quantify the relevance of each binding domain (color) to fold the structure of the Epha4 locus, in all the studied cases. To this aim, we measured how the distance-corrected Pearson correlation, $r^{\prime}$ (see below), between model and experimental Hi-C data, was affected when individual colors were withdrawn from the model (Supplementary Note). For example, we found that in mouse $\mathrm{CH} 12$ LX cells, no color is redundant, as $r^{\prime}$ was reduced in a range from $8 \%$ up to $15 \%$ of its original value. In particular, there is a subgroup of 6 main binding domains having an impact higher than the average (12\%). Analysis of the models in other murine and human cell types, in WT cells and mutants, gave similar results (Supplementary Note).

Each of the different $n^{*}$ types of binding domains is specified by the coordinates (in bases) of their binding sites along the locus. To quantify the similarity between pairs of binding domains (colors), we measured their genomic overlap, which turns out to be far from random (Supplementary Note). Analogously, the assignment of the binding domains to class I-II (see Results) is based on their overlaps with the locus TADs ${ }^{3}$ : specifically, a binding domain is of type I if it strongly overlaps (above median) only one or two consecutive TADs, else it is of type II (see Supplementary Note). Finally, to get some insights into the molecular nature of the inferred different types of binding sites, we correlated their genomic positions with epigenetic features available for CH12-LX cells in the ENCODE database ${ }^{24}$ (Supplementary Table 3). Specifically, for each binding domain we computed the Pearson correlation coefficient between the locations of its binding sites and the peaks of a chromatin mark, in the corresponding 10-kb bins. Next, to check the statistical significance of such correlations, we computed the distribution of correlations with chromatin marks of a random control model obtained by bootstrapping our binding domains. The correlation with a chromatin feature is considered significant if it is above the 95th or below the 5th percentile of the distribution of correlations in the random model. The resulting matrix of correlations is shown in Supplementary Fig. 3. We found that our binding domains correlated with distinct combinations of chromatin marks, rather than matching each with a single molecular factor.

Molecular dynamics simulations of the EPHA4 locus and its mutations. In our SBS model, the polymer beads and the binders are subject to a Brownian motion, described by the Langevin equation, numerically integrated with the Verlet algorithm in the LAMMPS package ${ }^{35,36}$. We set the diameters of polymer beads and of binders to $\sigma$, and their masses to $m$. The interaction potential used in our MD simulations has been developed in classical computer studies of polymer physics ${ }^{37}$. Two consecutive beads along the polymer are bound by a finitely extensible spring FENE potential ${ }^{37}$. To model excluded volume effects, the Weeks-ChandlerAndersen potential is used ${ }^{37}$. Finally, the binders interact with their cognate polymer beads via a short-ranged, attractive Lennard-Jones potential ${ }^{16}$. All details about the MD simulations are provided in the Supplementary Note.

The PRISMR polymer chain model of the WT Epha4 locus in murine CH12-LX cells (Fig. 2a), derived from published in situ Hi-C data at 10-kb resolution ${ }^{23}$, is made of $N=12,600$ beads. To model structural variants, we start from the WT polymer, implement the mutation under consideration, and perform new MD simulations. For instance, we removed the part of the polymer corresponding to the DNA deletion in real cells, or we inverted the binding sites corresponding to the experimental inversion. The resulting polymer models for the DelBs and DelB deletions and the $I n v F$ inversion are made respectively of $N=9,513$, $N=9,093$, and $N=12,600$ beads (Supplementary Fig. 5a). The models derived from our murine $\mathrm{E} 11.5$ cell $\mathrm{cHi}-\mathrm{C}$ data, our $\mathrm{CHiC}$ data of human skin fibroblast cells, and published human IMR90 cell data ${ }^{23}$ are analogous (Figs. $2 \mathrm{a}$ and $4 \mathrm{a}$, Supplementary Fig. 6a, and Supplementary Note). 
Polymer models including prior knowledge of CTCF binding sites. We considered a variant of our PRISMR model (PRISMR + CTCF) in which forward/ reverse CTCF binding sites are included; they are supposed to interact with an additional type of binder that can only bridge oppositely directed CTCF sites. We use peak-called CTCF ChIP-seq data ${ }^{38}$ and apply a standard motif finding analysis (using the FIMO tool in the MEME Suite online software version 4.12.0; see URLs) ${ }^{39}$ to obtain the best-matching peak in a $10-\mathrm{kb}$ window and its orientation (Supplementary Note). The CTCF motif ${ }^{40}$ was obtained from the JASPAR database (see URLs). Additionally, to try to dissect the specific effects of CTCF alone, we also considered a simpler polymer model (CTCF-only) including only the above described CTCF binding sites/binders, without the binding domains identified by the PRISMR model (Supplementary Note). The PRISMR + CTCF model does not significantly improve the comparison against $\mathrm{cHi}-\mathrm{C}$ data with respect to PRISMR alone (Supplementary Table 1), whereas the CTCF-only model fits some of the loops well, but has a poor distance-corrected Pearson correlation with $\mathrm{cHi}-\mathrm{C}$ data, $r^{\prime}=0.05$ (Supplementary Fig. 4).

Contact frequency matrices. The average pairwise contact frequency matrices of the polymer models are derived as discussed in the literature $e^{14,17}$. The distance $r_{i j}$ between any pair of beads $i$ and $j$ of the same type is measured in a given equilibrium polymer configuration; if $r_{i j}$ is lower than a set threshold, $i$ and $j$ are considered in contact. Averages are then taken over independent, single-molecule 3D structures (Supplementary Note).

To identify significant ectopic interactions, the normalized subtraction matrices (Figs. 3 b and 4 b and Supplementary Figs. 5b, 6b, 8, and 11), are computed. First, the matrix corresponding to the mutation is multiplied by a factor to equalize, in the regions not affected by the mutation, the reads count to the WT case, and then the WT matrix is subtracted from it. Next, to account for the distance bias, such a matrix is normalized by diving each subdiagonal by the average WT reads count at that genomic distance. Significant ectopic interactions correspond to absolute differences $>2$ s.d. (Supplementary Note)

To better highlight ectopic interactions, we produced virtual $4 \mathrm{C}$ plots from the viewpoint of the phenotype causative genes in each mutant in mouse (Fig. $3 \mathrm{c}$ and Supplementary Figs. 5c and 6c) and in human (Fig. 4c). Virtual 4C plots are obtained by plotting the column in the contact matrix corresponding to the considered viewpoint. To have a fair comparison between WT and mutation, we first normalized the WT matrix by equalizing the number of its reads to the total reads in the mutation, as described above.

To better compare experimental and model predicted contact matrices, we also measured the distance corrected Pearson correlation, $r^{\prime}$, i.e., the correlation after the effect of genomic distance is subtracted from a contact map (Supplementary Fig. 2). Specifically, we subtracted from each diagonal of the contact matrices (experimental and predicted) their average contact frequency at that genomic distance, and then calculated the Pearson correlation coefficient. The control case, matrices with bootstrapped diagonals, returns values of $r^{\prime}<0.05$ (Supplementary Note). Analogous comparisons of experimental replicates (see below and Supplementary Note) show that PRISMR versus $\mathrm{cHiC}$ correlations are comparable to those among replicates.

SureSelect design. We designed the SureSelect enrichment RNA probes over the genomic intervals chr1: 71,000,001-81,000,000 (mm9) and chr2: 218,314,001224,093,000 (hg19) using SureDesign from Agilent. Probes were not specifically designed in proximity of DpnII sites but over the entire region, with a coverage of $85 \%$.

Mouse samples. Mouse embryonic stem (ES) cell lines were established from DelB and DelBs homozygous blastocysts using N2B27 Medium supplemented with FGF/Erk and Gsk3 pathway inhibitors (2i) and LIF ${ }^{41}$. Both mouse strains (DelB and DelBs) were previously maintained by crossing them with C57BL6/J mice. The derived ES cells, in addition to the preexisting wild type G4 and InvF homozygous cell lines $(129 / \mathrm{Sv} \times \mathrm{C} 57 \mathrm{BL} / 6 \mathrm{~F} 1 \mathrm{hybrid})^{7}$, were tested for mycoplasma contamination using Mycoalert detection kit (Lonza, catalog number LT07-118) and Mycoalert assay control set (Lonza, catalog number LT07-518). These ES cells lines were used to generate homozygous embryonic litters using tetraploid complementation $^{42}$. All animal procedures were conducted as approved by the local authorities (LAGeSo Berlin) under license numbers G0368/08 and G0247/13.

Human materials. Skin biopsies and were obtained from the patients and controls by standard procedures. Fibroblasts were cultured in DMEM (Lonza) supplemented with 10\% FCS (Gibco), 1\% L-glutamine (Lonza), and 1\% penicillin/ streptomycin (Lonza). Cells were tested for mycoplasma contamination as described above. Written informed consent was obtained from all individuals studied to participate in this study. This study was approved by the Charité Universitätsmedizin Berlin ethics committee and complies with all relevant ethical regulations.

cHi-C. Libraries were prepared from mouse E11.5 mutant and wild-type distal fore- and hindlimbs and from human cultured skin fibroblasts from patients and healthy controls. as previously described ${ }^{43}$, i.e. crosslinking, cell lysis, DpnII digestion, ligation, and de-crosslinking. DNA was then sheared with a Covaris sonicator (duty cycle: $10 \%$, intensity: 5 , cycles per burst: 200 , time: 6 cycles of 60 s each, set mode: frequency sweeping, temperature: $4^{\circ}$ to $7^{\circ} \mathrm{C}$ ). Adaptors were ligated to the sonicated DNA, which was then amplified according to Agilent instructions. The library was then hybridized to SureSelect RNA probes, indexed for sequencing following Agilent instructions and sequenced using a 100-bp paired-end mode.

Capture Hi-C experiments were performed as singletons. As an internal control, we compared the results from all four experiments for regions outside of the region of interest (for mouse: chr1: 71,000,001-74,820,000 and chr1: 78,080,001-81,000,000; for human: chr2: 218,320,001-219,730,000 and chr2: $223,030,001-224,090,000)$. The $\mathrm{cHi}-\mathrm{C}$ maps of the internal control were highly correlated between the four samples (mouse samples Spearman $r$ : WT/DelB $=0.99$; $\mathrm{WT} / \operatorname{DelBs}=0.99 ; \mathrm{WT} / \operatorname{InvF}=0.99 ; \operatorname{DelB} / \operatorname{DelBs}=0.99 ; \operatorname{DelB} / \operatorname{InvF}=0.99$; DelBs $/ \operatorname{InvF}=0.99$; human samples Spearman $r$ WT $/$ DelB $=0.99$; WT $/ D u p F=0.98$; $\mathrm{WT} / \mathrm{Dup} P=0.98 ; \mathrm{DelB} / \mathrm{DupF}=0.99 ; \mathrm{DelB} / \mathrm{DupP}=0.98 ; \mathrm{DupF} / \mathrm{DupP}=0.98)$, confirming the high reproducibility of the methodology.

cHi-C data processing. DNA libraries were sequenced paired-end. Fastq files were processed with the HiCUP pipeline v0.5. $8^{44}$ (nofill: 1, format: Sanger, without di-tag length restriction) to perform the mapping as well as to filter for valid and unique di-tags. The pipeline was set up with Bowtie2 v2.2.6 $6^{45}$ and with reference genomes hg19 and mm9, respectively. Additionally, we created customized genome files containing the mutation for the SVs investigated in mouse (Supplementary Table 4). BAM files produced by the HiCUP pipeline were transferred into an intermediate text file format suited for Juicebox command line tools ${ }^{23,46}$, which were used for binning and normalization (KR normalization ${ }^{33}$ ). The genomic interval enriched by the $\mathrm{cHi}-\mathrm{C}$ protocol covers $5.77 \mathrm{Mb}$ in hg19 and $10 \mathrm{Mb}$ in $\mathrm{mm} 9$, respectively, leading to three different regimes in the contact map: (i) enriched vs. enriched, (ii) enriched vs. nonenriched, and (iii) nonenriched vs. nonenriched. For binning and normalization, only regime (i) was considered. Therefore, di-tags were filtered for the enriched genomic interval and all coordinates were shifted by the start of the enriched interval (Supplementary Table 4). The chromosome size file for Juicebox command line tools was customized such that it only contained the size of the enriched region. The minimum MAPQ for the import was set to 30. After binning and normalization, all coordinates were shifted back to their original values. The applied KR (Knights and Ruiz) normalization ${ }^{33}$ is a matrixbalancing algorithm that ensures equal sums for all rows and columns of the map. The underlying assumption for this type of normalization is that all loci should have an equal representation in the map. When SVs, such as duplications, are mapped to a WT reference genome, as well as at the map borders, the map deviates strongly from this assumption and matrix balancing corrects disproportionally. Therefore, only raw count maps were used to create subtraction maps between WT and SVs (see section "Contact frequency matrices"). In all cases, cHi-C data confirmed the presence of the corresponding SVs on each mouse and human studied sample.

Statistics. No statistical methods were used to predetermine sample size. There was no exclusion/inclusion of samples or animals in the analysis, no randomization of experiments, and investigators were not blinded during experiments and outcome assessment. No unique materials were used in this study.

All the statistical tests we used are described in the paper and all details provided in the Methods and Supplementary Note. We used Pearson correlation coefficients to compare experimental and model derived contact matrices. Pearson correlations were also used to match the model-inferred binding domains with epigenetic features from $\mathrm{ENCODE}^{24}$. One-tailed Wilcoxon's rank-sum tests were applied to check the significance of the binding domains.

Reporting Summary. Further information on experimental design is available in the Nature Research Reporting Summary linked to this article.

Code availability. We used publicly available computer codes (LAMMPS) for our molecular dynamics simulations. Custom codes used to generate results reported in the manuscript can be made available upon request. All details of the algorithms are illustrated above and in previous publications cited herein.

\section{Data availability. Data have been deposited at GEO under accession code} GSE92294.

URLs. FIMO: http://meme-suite.org/tools/fimo; JASPAR database, http://jaspar. binf.ku.dk/; Gene Expression Omnibus, https://www.ncbi.nlm.nih.gov/geo/.

\section{References}

30. de Gennes, P. G. Scaling Concepts in Polymer Physics (Cornell Univ. Press, Ithaca, NY, 1979).

31. Kirkpatrick, S., Gelatt, C. D. Jr \& Vecchi, M. P. Optimization by simulated annealing. Science 220, 671-680 (1983).

32. Salamon, P., Sibani, P. \& Frost, R. Facts, Conjectures, and Improvements for Simulated Annealing (SIAM, Philadelphia, 2002). 
33. Knight, P. A. \& Ruiz, D. A fast algorithm for matrix balancing. IMA J. Numer. Anal. 33, 1029-1047 (2013).

34. Parisi, G. Statistical Field Theory (Westview Press, New York, 1998).

35. Rosa, A. \& Everaers, R. Structure and dynamics of interphase chromosomes. PLOS Comput. Biol. 4, e1000153 (2008).

36. Plimpton, S. Fast parallel algorithms for short-range molecular dynamics. J. Comput. Phys. 117, 1-19 (1995).

37. Kremer, K. \& Grest, G. S. Dynamics of entangled linear polymer melts: A molecular-dynamics simulation. J. Chem. Phys. 92, 5057 (1990).

38. Andrey, G. et al. Characterization of hundreds of regulatory landscapes in developing limbs reveals two regimes of chromatin folding. Genome Res. 27, 223-233 (2017).

39. Grant, C. E., Bailey, T. L. \& Noble, W. S. FIMO: scanning for occurrences of a given motif. Bioinformatics 27, 1017-1018 (2011).
40. Barski, A. et al. High-resolution profiling of histone methylations in the human genome. Cell 129, 823-837 (2007).

41. Nagy, K. N. J. in Advanced Protocols for Animal Transgenesis (eds. Pease, S. \& Saunders, T. L.) 431-455 (Springer, Berlin, 2011).

42. Artus, J. \& Hadjantonakis, A. K. Generation of chimeras by aggregation of embryonic stem cells with diploid or tetraploid mouse embryos. Methods Mol. Biol. 693, 37-56 (2011).

43. Hagège, $\mathrm{H}$. et al. Quantitative analysis of chromosome conformation capture assays (3C-qPCR). Nat. Protoc. 2, 1722-1733 (2007).

44. Wingett, S. et al. HiCUP: pipeline for mapping and processing Hi-C data. F1000Res. 4, 1310 (2015).

45. Langmead, B. \& Salzberg, S. L. Fast gapped-read alignment with Bowtie 2. Nat. Methods 9, 357-359 (2012).

46. Durand, N. C. et al. Juicer provides a one-click system for analyzing loop-resolution Hi-C experiments. Cell Syst. 3, 95-98 (2016). 


\section{natureresearch}

\section{Life Sciences Reporting Summary}

Nature Research wishes to improve the reproducibility of the work that we publish. This form is intended for publication with all accepted life science papers and provides structure for consistency and transparency in reporting. Every life science submission will use this form; some list items might not apply to an individual manuscript, but all fields must be completed for clarity.

For further information on the points included in this form, see Reporting Life Sciences Research. For further information on Nature Research policies, including our data availability policy, see Authors \& Referees and the Editorial Policy Checklist.

Please do not complete any field with "not applicable" or n/a. Refer to the help text for what text to use if an item is not relevant to your study. For final submission: please carefully check your responses for accuracy; you will not be able to make changes later.

\section{- Experimental design}

\section{Sample size}

Describe how sample size was determined.

\section{Data exclusions}

Describe any data exclusions.

\section{Replication}

Describe the measures taken to verify the reproducibility of the experimental findings.

\section{Randomization}

Describe how samples/organisms/participants were allocated into experimental groups.

\section{Blinding}

Describe whether the investigators were blinded to group allocation during data collection and/or analysis.
Capture $\mathrm{Hi}-\mathrm{C}$ experiments are extremely reproducible among biological replicates when samples are processed appropriately. For this reason, experiments for each condition are performed only once $(n=1)$. To control that experimental procedures are reproducible, samples from different experiments were compared. In this comparison, control genomic regions that are unaffected by structural variants (and therefore should show the same interaction profile) are compared. Such comparison shows that samples are highly reproducible from a technical point of view (see Methods, "CHiC" subsection, 2nd paragraph).

There was no exclusion/inclusion of samples or animals in the analysis.

To control that Capture Hi-C experimental procedures are reproducible, samples from different experiments were compared. In this comparison, control genomic regions that are unaffected by structural variants (and therefore should show the same interaction profile) are compared. Such comparison showed that samples are highly reproducible from a technical point of view (see Methods, "CHiC" subsection, 2nd paragraph). All experimental attempts were successful

There was no randomization of experiments.

Investigators were not blinded during experiments and outcome assessment.

Note: all in vivo studies must report how sample size was determined and whether blinding and randomization were used. 


\section{Statistical parameters}

For all figures and tables that use statistical methods, confirm that the following items are present in relevant figure legends (or in the Methods section if additional space is needed).

n/a $\mid$ Confirmed

$\bigotimes$ The exact sample size $(n)$ for each experimental group/condition, given as a discrete number and unit of measurement (animals, litters, cultures, etc.)

$\checkmark$ A description of how samples were collected, noting whether measurements were taken from distinct samples or whether the same sample was measured repeatedly

$\bigotimes$ A statement indicating how many times each experiment was replicated

$\varnothing$ The statistical test(s) used and whether they are one- or two-sided

Only common tests should be described solely by name; describe more complex techniques in the Methods section.

Х $\square$ A description of any assumptions or corrections, such as an adjustment for multiple comparisons

Test values indicating whether an effect is present

Provide confidence intervals or give results of significance tests (e.g. P values) as exact values whenever appropriate and with effect sizes noted.

$\bigotimes$ A clear description of statistics including central tendency (e.g. median, mean) and variation (e.g. standard deviation, interquartile range)

$\triangle \square$ Clearly defined error bars in all relevant figure captions (with explicit mention of central tendency and variation)

See the web collection on statistics for biologists for further resources and guidance.

\section{- Software}

Policy information about availability of computer code

\section{Software}

Describe the software used to analyze the data in this study.

We used a standard motif finding analysis (using the FIMO tool in the MEME Suite online software version 4.12.0).

Fastq files were processed with the HiCUP pipeline v0.5.843 (Nofill:1, Format: Sanger, without di-tag length restriction) performing the mapping as well the filtering for valid and unique di-tags. The pipeline was set up with Bowtie2 v2.2.644 .

We used publicly available computer codes (LAMMPS) for our Molecular Dynamics simulations. Custom codes used to generate results reported in the manuscript will be made available upon request. All details of the algorithms are illustrated in the Methods section and in previous publications cited therein

For manuscripts utilizing custom algorithms or software that are central to the paper but not yet described in the published literature, software must be made available to editors and reviewers upon request. We strongly encourage code deposition in a community repository (e.g. GitHub). Nature Methods guidance for providing algorithms and software for publication provides further information on this topic.

\section{- Materials and reagents}

Policy information about availability of materials

\section{Materials availability}

Indicate whether there are restrictions on availability of unique materials or if these materials are only available for distribution by a third party.

\section{Antibodies}

Describe the antibodies used and how they were validated for use in the system under study (i.e. assay and species).
No unique materials were used in this study.

No antibodies were used in this study. 
10. Eukaryotic cell lines

a. State the source of each eukaryotic cell line used.

b. Describe the method of cell line authentication used.

c. Report whether the cell lines were tested for mycoplasma contamination.

d. If any of the cell lines used are listed in the database of commonly misidentified cell lines maintained by ICLAC, provide a scientific rationale for their use.
Mouse ES cell lines were established from DelB and DelBs homozygous blastocysts using N2B27 Medium supplemented with FGF/Erk and Gsk3 pathway inhibitors (2i) and LIF. Both mouse strains (DelB and DelBs) were previously maintained by crossing them with C57BL6/J mice. The derived ES cells, in addition to the preexisting wild type G4 (129/Sv × C57BL/6 F1 hybrid; kindly provided by Andras Nagy's lab) and InvF homozygous cell lines (129/Sv $\times$ C57BL/6 F1 hybrid, generated through CRISPR genome editing from G4 cells; reported in Lupiáñez et al., 2015) were used to generate homozygous embryonic litters using tetraploid complementation.

Skin biopsies and were obtained from the patients and controls by standard procedures. Fibroblasts were cultured in DMEM (Lonza) supplemented with 10\% fetal calf serum (Gibco), $1 \%$ I-glutamine (Lonza) and 1\% penicillin/streptomycin (Lonza).

In all cases, $\mathrm{CHi}-\mathrm{C}$ data confirmed the presence of the corresponding SVs on each mouse and human studied sample.

All cell lines tested negative for mycoplasma contamination.

No commonly misidentied cell lines were used.

\section{- Animals and human research participants}

Policy information about studies involving animals; when reporting animal research, follow the ARRIVE guidelines

\section{Description of research animals}

Provide all relevant details on animals and/or animal-derived materials used in the study.
Mouse ES cell lines (129/Sv × C57BL/6 F1 hybrid background and 129/Sv × C57BL/6 F1 hybrid background backcrossed with $\mathrm{C} 57 \mathrm{BL} / 6$ ) were used to generate homozygous embryonic litters using tetraploid complementation. Resulting embryos of both sexes were analyzed at E 11.5

Policy information about studies involving human research participants

\section{Description of human research participants}

Describe the covariate-relevant population characteristics of the human research participants.
Sex and gender from patients and controls are heterogeneous but not relevant for the results of the study as Capture $\mathrm{Hi}-\mathrm{C}$ experiments are not influenced by those parameters 